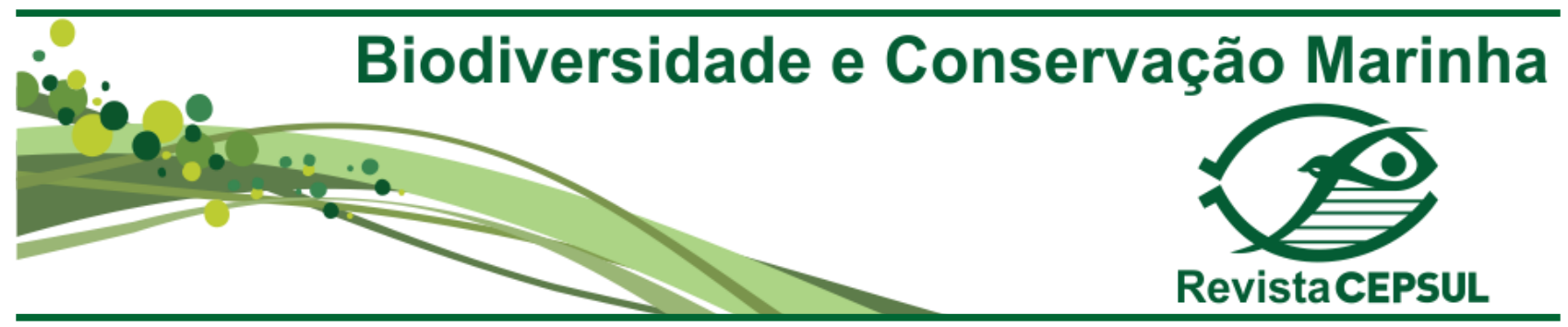

\title{
Recursos-alvo que são também bycatch, e recomendação para a gestão da pesca de emalhe no litoral do Paraná, Brasil
}

\author{
Paulo de Tarso da Cunha Chaves ${ }^{1} \&$ Amanda Ventura Firmino da Silva ${ }^{1}$
}

${ }^{1}$ Departamento de Zoologia, Universidade Federal do Paraná - UFPR, Caixa Postal 19020, CEP - 81531-98o, Curitiba, PR, Brasil, ptchaves@ufpr.br,vfs.amanda@gmail.com.

Submetido em: 11/12/2017; Aceito em: 26/10/2018; Publicado em: 03/o1/2019

Resumo. Estudou-se a captura não-alvo, em redes de emalhe na pesca de pequena escala no litoral do Paraná. De março a outubro de 2017 foram observados na Praia Central de Matinhos 28 desembarques pesqueiros, colhendo-se informações sobre técnicas de captura, dimensões das redes, recursos-alvo e acompanhantes, e destinação dos pescados. Trinta e três gêneros de pescados foram identificados. Cavala foi o recurso mais comum na condição de espécie-alvo, e peixe-espada e corvina, de fauna acompanhante. Todos os recursos-alvo ocorreram ao menos uma vez na condição não-alvo, corvina o mais comum. Tal captura, aqui genericamente designada incidental, foi mais variada na técnica fundeio, que visa a recursos demersais, que na caceio, que visa a pelágicos, mas ambas apresentaram captura incidental demersal e pelágica, indistintamente. Parte deste bycatch é comercializada ou doada, outra é descartada ao mar. A normatização da pesca com emalhe deve considerar que a diversidade da fauna em regiões tropicais amplia o espectro de capturas não desejadas ou, até, inconvenientes. Visto que toda espécie-alvo pode ocorrer também como bycatch, normas de pesca baseadas em especificações das redes não são efetivas para a conservação dos recursos na região estudada, podendo a exclusão temporária de áreas de pesca, mediante rodízio, ser melhor instrumento de manejo.

Palavras-chave: Captura incidental; recursos-alvo; Scomberomorus; Micropogonias; Cynoscion; caceio; fundeio.

\begin{abstract}
Target resource occurring as bycatch, with advices on management of gillnet fisheries in Southern Brazil. Incidental catches of gillnet fisheries were studied in the small-scale fisheries performed at Parana coast, Southern Brazil. From March to October 2017 twenty-eight landings were observed, giving data on fishery techniques, gillnet dimensions, target resource and bycatch, and catches use. Thirty-three genera were identified. Serra Spanish mackerel (Scombridae) was the most common target resource, and largehead hairtail (Trichiuridae) and whitemouth croaker (Sciaenidae) were the most common bycatch. All target resources, mainly the whitemouth croaker, have occurred also as
\end{abstract}


bycatch. These catches, hence designed incidental ones, were more diversified in the fundeio technique, that aims demersal resources, than in the caceio one, that aims pelagic resources. Caceio and fundeio have presented, both of them, demersal and pelagic bycatches. Part of bycatch is commercialized or donated, and part is discarded aboard. Fishery rules for gillnet fishery should consider that the fauna diversity in tropical zones increases catches of non target species. Considering that every target species can also be a bycatch, fisheries rules based on gillnet specifications are not effective for conservation of resources at the studied area. Temporary, rotative closing areas for exploitation would be considered as a better management tool.

Keywords. incidental catch; target resources; Scomberomorus; Micropogonias; Cynoscion, caceio and fundeio.

\section{Introdução}

Pesca incidental pode ser conceituada como a captura de organismos diferentes do alvo da pescaria, compondo um conjunto coletivamente denominado fauna acompanhante (Alverson et al., 1994). No Brasil, a legislação (Brasil, 2011) denomina fauna acompanhante previsível a captura não-alvo passível de comercialização, e restringe captura incidental àquela de captura interditada, não passível de comercialização. Genericamente a captura incidental é conhecida como bycatch, mas inexiste consenso, podendo ele corresponder aos recursos que não serão comercializados, seja por falta de interesse econômico, seja por força legal (NOAA, 2007). Fato é que a coexistência espaço-temporal entre recursos-alvo e nãoalvo, comum na elevada diversidade das regiões tropicais, eleva o espectro de espécies capturadas. Na pesca de pequena escala a fauna acompanhante apresenta valor comercial e tem aproveitamento, diferente do que ocorre na industrial, em que o descarte é maior (Eayrs, 2007). Impactos negativos da captura incidental são, por exemplo, captura de indivíduos juvenis, ou prestes a reproduzir-se; em tamanho fora do autorizado na legislação; ou de espécies com captura proibida. Espécies ameaçadas, em perigo ou protegidas são, assim, expostas. Longlines pelágicos incidem sobre aves e tartarugas marinhas, e, junto a redes de variados tipos, constituem a principal causa de mortalidade de mamíferos marinhos, número que mun- dialmente alcança 665 mil indivíduos (Lenta \& Squires, 2017). Animais capturados incidentalmente também afetam equipamentos de pesca, pelo corte de cabos e redes, e danos em armadilhas. $\mathrm{O}$ fato gera em pescadores profissionais certa repulsa contra botos e pinguins, por exemplo, frequentemente emalhados em redes (Cardoso et al., 2011).

O ordenamento pesqueiro define esforço, cotas e permissões, época, espaço geográfico, petrechos, e toma por referência os recursos -alvo. No caso do emalhe, em particular, recomendações para ordenamento (IBAMA/ CEPSUL, 2006) já incluíram dimensões dos petrechos e distâncias de afastamento entre eles, áreas de exclusão para a frota industrial, emissão das permissões de pesca, e defeso quanto a recursos em particular. A captura incidental é lembrada, porém atentando-se, apenas, a espécies ameaçadas, como tartarugas, mamíferos, meros e elasmobrânquios. A eleição de "espécies-bandeira" (IBAMA/CEPSUL, 2006) para referenciar o ordenamento parece omitir que também recursos cuja captura é permitida e o comércio, atraente, estão vulneráveis quando, na condição de fauna acompanhante, são capturados em tamanho ou quantidade incompatíveis com sua capacidade de reposição.

Capturas que diferem dos recursos-alvo são comuns na pesca marítima dos Estados do Paraná e Santa Catarina, tanto na pesca de pequena escala (Souza \& Chaves, 2007; Pina \& Chaves, 2009), como na industrial (Schroeder 
et al., 2014). Na de pequena escala paranaense, a frota atua com redes de emalhe voltadas a peixes e camarão-branco, Litopenaeus schmitti, e com arrasto de portas voltado ao camarãosete-barbas, Xiphopenaeus kroyeri. O emalhe é praticado mediante técnicas de fundeio, caceio, cerco e caracol, variando com alvos e sazonalidade (Chaves \& Robert, 2003; Afonso, 2016). O presente trabalho relata a captura de recursos pesqueiros não-alvo da pesca com emalhe no litoral sul do Paraná, informando sua composição, segundo recurso-alvo, e o destino que lhe é dado. Os resultados podem subsidiar ações para maior eficácia no ordenamento da pesca com redes de emalhe na região.

\section{Materiais e métodos}

De março a outubro de 2017 acompanhou-se o desembarque da pesca com emalhe na Praia Central do município de Matinhos em oito datas. Nesse período, o esforço de campo foi heterogêneo: dados foram colhidos de 28 desembarques, cinco no primeiro quadrimestre e 23 no segundo. A tomada de dados deu-se por visualização do material a bordo e por anotação de informações fornecidas pelos pescadores. Registraram-se dados da pescaria em 24 dos desembarques, pois nos demais apenas o recurso desembarcado foi acessado. Tais dados foram: técnica de pesca, altura da rede, tempo de espera para retirada das redes, recurso-alvo, fauna acompanhante e destino do descarte.

Neste trabalho será adotada a expressão bycatch para designar todos os recursos pesqueiros capturados sem que fossem o alvo da pescaria, portanto em caráter incidental ou de fauna acompanhante, independentemente de restrições legais ou econômicas.

Recursos-alvo e bycatch foram identificados ao menor nível possível (espécie ou gênero). O alvo da pescaria era informado pelos pescadores e seu nome comum, anotado em planilha. Por tal limitação nomenclatural, certos alvos foram tratados no nível de gênero, apenas (ex.: o alvo cavalas foi reconhecido como Scomberomorus spp).

A frequência de ocorrência de cada re- curso foi mensurada dividindo-se o número total de registros na condição em questão alvo ou bycatch - pela soma das frequências do conjunto de recursos na mesma condição. Os resultados são referenciados em dois grupamentos sazonais: final de verão e outono, correspondendo às observações de março a junho, e inverno e início de primavera, de julho a outubro.

\section{Resultados}

A maior parte dos pescados é comercializada no Mercado Municipal da cidade de Matinhos. Os pescadores, normalmente uma dupla por canoa, desembarcam em caixas plásticas peixes e camarões, majoritariamente já selecionados para processamento e comércio. $\mathrm{O}$ bycatch é aproveitado parcialmente: parte é doada aos cambistas, pessoas que informalmente ajudam no recolhimento das embarcações para terra; parte segue para comercialização, seja a baixo custo, os indivíduos de pequeno porte na categoria 'mistura', seja a custo maior, os peixes e camarões que alcançam bom valor comercial; e parte é descartada.

No conjunto de desembarques, em cada um deles somando-se observações dos autores a depoimentos de pescadores, 33 recursos foram registrados (Tabela 1). Destes, seis eram alvo, sendo cavala, Scomberomorus spp, o mais frequente, presente em $50 \%$ dos desembarques. Outros alvos foram pescada, Cynoscion spp (28,6\% dos desembarques); camarãopistola, Litopenaeus schmitti $(7,1 \%)$; corvina, Micropogonias furnieri (7,1\%); linguado, $\mathrm{Pa}$ ralichthys spp $(3,6)$; e robalo, Centropomus spp (3,6\%) (Tabela 2). Na condição de bycatch, os recursos presentes em mais desembarques foram peixe-espada, Trichiurus lepturus, e corvina $(10,7 \%)$, pescada $(8,2 \%)$, robalo $(7,4 \%)$, betara, Menticirrhus spp (6,6\%), e peixeporco, Balistes capriscus (5,7\%). Outros acompanhantes representaram menos que $5 \%$ da frequência total (Tabela 2).

Constatou-se que um recurso pode estar na condição de alvo numa pescaria e acompanhante noutra. Todos os alvos foram encontrados como bycatch em pelo menos um desem- 
Tabela 1. Identificação dos recursos registrados de março a outubro de 2017 no desembarque de 28 embarcações pesca com emalhe em Matinhos-PR.

\begin{tabular}{|c|c|c|c|}
\hline Grupo & Família & Nome popular & Identificação científica \\
\hline \multirow{5}{*}{ Elasmobranchii } & Carcharhinidae & Cação rola-rola & Rhizoprionodon spp \\
\hline & Myliobatidae & Raia-cachorro & Rhinoptera spp \\
\hline & \multirow{2}{*}{ Rhinobatidae } & Raia-emplastro & Zapteryx brevirostris \\
\hline & & Raia-viola & Rhinobatos spp \\
\hline & Sphyrnidae & Tubarão-martelo & Sphyrna spp \\
\hline \multirow{24}{*}{ Actinopterygii } & Ariidae & Bagre & Genidens spp \\
\hline & Balistidae & Peixe-porco & Balistes capriscus \\
\hline & \multirow{2}{*}{ Carangidae } & Peixe-galo & Selene spp \\
\hline & & Salteira & Oligoplites spp \\
\hline & Centropomidae & Robalo & Centropomus spp \\
\hline & Clupeidae & Sardinha & Sardinella brasiliensis \\
\hline & Dactylopteridae & Peixe-voador & Dactylopterus volitans \\
\hline & \multirow[t]{2}{*}{ Elopidae } & Ubarana & Elops saurus \\
\hline & & Carapeba & Eugerres brasilianus \\
\hline & \multirow[t]{2}{*}{ Gerreidae } & Caratinga & Diapterus rhombeus \\
\hline & & Escrivão & Encinostomus sp \\
\hline & Lutjanidae & Vermelho & Lutjanus spp \\
\hline & Mugilidae & Tainha & Mugil liza \\
\hline & Paralichthyidae & Linguado & Paralichthys spp \\
\hline & Pomatomidae & Anchova & Pomatomus saltatrix \\
\hline & \multirow{6}{*}{ Sciaenidae } & Betara & Menticirrhus spp \\
\hline & & Cangulo & Stellifer spp \\
\hline & & Corvina & Micropogonias furnieri \\
\hline & & Maria-luiza & Paralonchurus brasiliensis \\
\hline & & Oveva & Larimus breviceps \\
\hline & & Pescada & Cynoscion spp \\
\hline & Scombridae & Cavala & Scomberomorus spp \\
\hline & Stromateidae & Gordinho & Peprilus paru \\
\hline & Trichiuridae & Peixe-espada & Trichiurus lepturus \\
\hline Cephalopoda & Loliginidae & Lula & Lolliguncula spp \\
\hline \multirow{3}{*}{ Decapoda } & \multirow{2}{*}{ Penaeidae } & Camarão-pistola* & Litopenaeus schmitti \\
\hline & & Camarão-sete-barbas & Xiphopenaeus kroyeri \\
\hline & Portunidae & Siri-azul & Callinectes danae \\
\hline
\end{tabular}

${ }^{*}$ ) Camarão pistola: denominação local para camarão branco.

barque, sendo corvina o mais frequente e cavala, o menos. Vinte e sete recursos desembarcados não eram alvo das pescarias, com destaque ao peixe-espada, 10,7\% do bycatch, betara, 6,6\%, peixe-porco, $5,7 \%$, e cações Rhizoprionodon, 4,9\% (Tabela 2).

Os 24 desembarques com técnica de pesca identificada vinculavam-se ou a caceio, 12 operações, panos com altura de até 21 metros, ou a fundeio, 12 operações, panos de até 25 metros. As pescarias de caceio tinham como alvo cavala; as de fundeio, os demais recursosalvo, pescada principalmente, e corvina, cama- rão-pistola, linguado, robalo e cavala (Figura 1).

A pesca de caceio dirigida a recurso pelágico, tendo como alvo cavala, incidiu também sobre recursos demersais. Nesta pescaria o bycatch mais comum, registrado nos 14 desembarques acompanhados, compôs-se de peixeespada, corvina e peixe-porco, apenas o primeiro pelágico. Por outro lado, nas pescarias de fundeio com alvo demersal o bycatch compôs-se principalmente de recursos igualmente demersais: no alvo pescada, houve bycatch de betara, robalo e camarão-pistola, registrados 
Tabela 2. Pescados registrados na condição de alvo ou bycatch em 28 desembarques de emalhe de março a outubro de 2017, em Matinhos-PR. \%: frequência relativa ao número de desembarques em tal condição, todos os recursos somados. Indica-se hábito de vida, segundo literatura.

\begin{tabular}{|c|c|c|c|c|}
\hline Recurso & Alvo (\%) & Bycatch (\%) & Hábito & Referência para hábito \\
\hline Corvina & 7,1 & 10,7 & Demersal & Mulato et al. (2015) \\
\hline Peixe-espada & 0,0 & 10,7 & Pelágico & Barbanti et al. (2013) \\
\hline Pescada & 28,6 & 8,2 & Demersal & Magro et al. (2000) \\
\hline Robalo & 3,6 & 7,4 & Demersal & Barbanti et al. (2013) \\
\hline Betara & 0,0 & 6,6 & Demersal & Barbanti et al. (2013) \\
\hline Peixe-porco & 0,0 & 5,7 & Demersal & Magro et al. (2000) \\
\hline Cação-rola-rola & 0,0 & 4,9 & Demersal & $\begin{array}{l}\text { Rocha \& Rossi-Wongtschowski } \\
\text { (1998) }\end{array}$ \\
\hline Cangulo & 0,0 & 4,1 & Demersal & Barbanti et al. (2013) \\
\hline Gordinho & 0,0 & 4,1 & Pelágico & Barbanti et al. (2013) \\
\hline Vermelho & 0,0 & 4,1 & Demersal & Feitoza et al. (2005) \\
\hline Linguado & 3,6 & 3,3 & Demersal & $\begin{array}{l}\text { Rocha \& Rossi-Wongtschowski } \\
\text { (1998) }\end{array}$ \\
\hline Camarão-pistola & 7,1 & 2,5 & Demersal & Santos et al. (2004) \\
\hline Bagre & 0,0 & 2,5 & Demersal & Barbanti et al. (2013) \\
\hline Oveva & 0,0 & 2,5 & Demersal & Barbanti et al. (2013) \\
\hline Raias (3 espécies) & 0,0 & 2,5 & Demersal & Fagundes-Netto et al. (2011) \\
\hline Salteira & 0,0 & 2,5 & Pelágico & Barbanti et al. (2013) \\
\hline Cavala & 50,0 & 1,6 & Pelágico & Barbanti et al. (2013) \\
\hline Anchova & 0,0 & 1,6 & Pelágico & Haimovici et al. (2006) \\
\hline Lula & 0,0 & 1,6 & Demersal & Zaleski (2010) \\
\hline Peixe-voador & 0,0 & 1,6 & Demersal & $\begin{array}{l}\text { Rocha \& Rossi-Wongtschowski } \\
\text { (1998) }\end{array}$ \\
\hline Sardinha & 0,0 & 1,6 & Pelágico & Paiva \& Motta (2000) \\
\hline Siri-azul & 0,0 & 1,6 & Demersal & Chalegre (2008) \\
\hline Maria-luiza & 0,0 & 1,6 & Demersal & $\begin{array}{l}\text { Rocha \& Rossi-Wongtschowski } \\
\text { (1998) }\end{array}$ \\
\hline Ubarana & 0,0 & 0,8 & Pelágico & Fagundes-Netto et al. (2011) \\
\hline Camarão-sete-barbas & 0,0 & 0,8 & Demersal & Guimarães (2009) \\
\hline Carapeba & 0,0 & 0,8 & Demersal & Barbanti et al. (2013) \\
\hline Caratinga & 0,0 & 0,8 & Demersal & Barbanti et al. (2013) \\
\hline Escrivão & 0,0 & 0,8 & Demersal & Barbanti et al. (2013) \\
\hline Peixe-galo & 0,0 & 0,8 & Demersal & Menezes \& Figueiredo (1980) \\
\hline Tainha & 0,0 & 0,8 & Pelágico & Barbanti et al. (2013) \\
\hline Tubarão-martelo & 0,0 & 0,8 & Pelágico & Cortés et al. (2010) \\
\hline TOTAL & 100,0 & 100,0 & & \\
\hline
\end{tabular}

nos oito desembarques; no alvo corvina, bycatch linguado e gordinho, Peprilus paru, este pelágico, ambos em dois desembarques; no alvo robalo, bycatch gordinho e tainha, $\mathrm{Mu}$ gil liza (pelágicos), e, os demais, demersais, um desembarque; no alvo linguado, bycatch corvina e cação-rola-rola, Rhizoprionodon spp, um desembarque; e no alvo camarão-pistola, bycatch cangulo, Stellifer spp, e lula, Lolliguncula spp, recursos demersais, dois desembarques acompanhados (Tabela 3).
Fundeio foi a técnica com maior número de gêneros registrados na condição bycatch. Os mais frequentes foram corvina, pescada e betara. O caceio apresentou menor número de gêneros como bycatch, sendo os mais frequentes peixe-espada, corvina e peixe-porco (Tabela 4).

De março a junho foram registrados $45 \%$ dos 'recursos-alvo + bycatch' do período total de estudo; de julho a outubro, $84 \%$. A diferença de proporção entre quadrimestres, cer- 


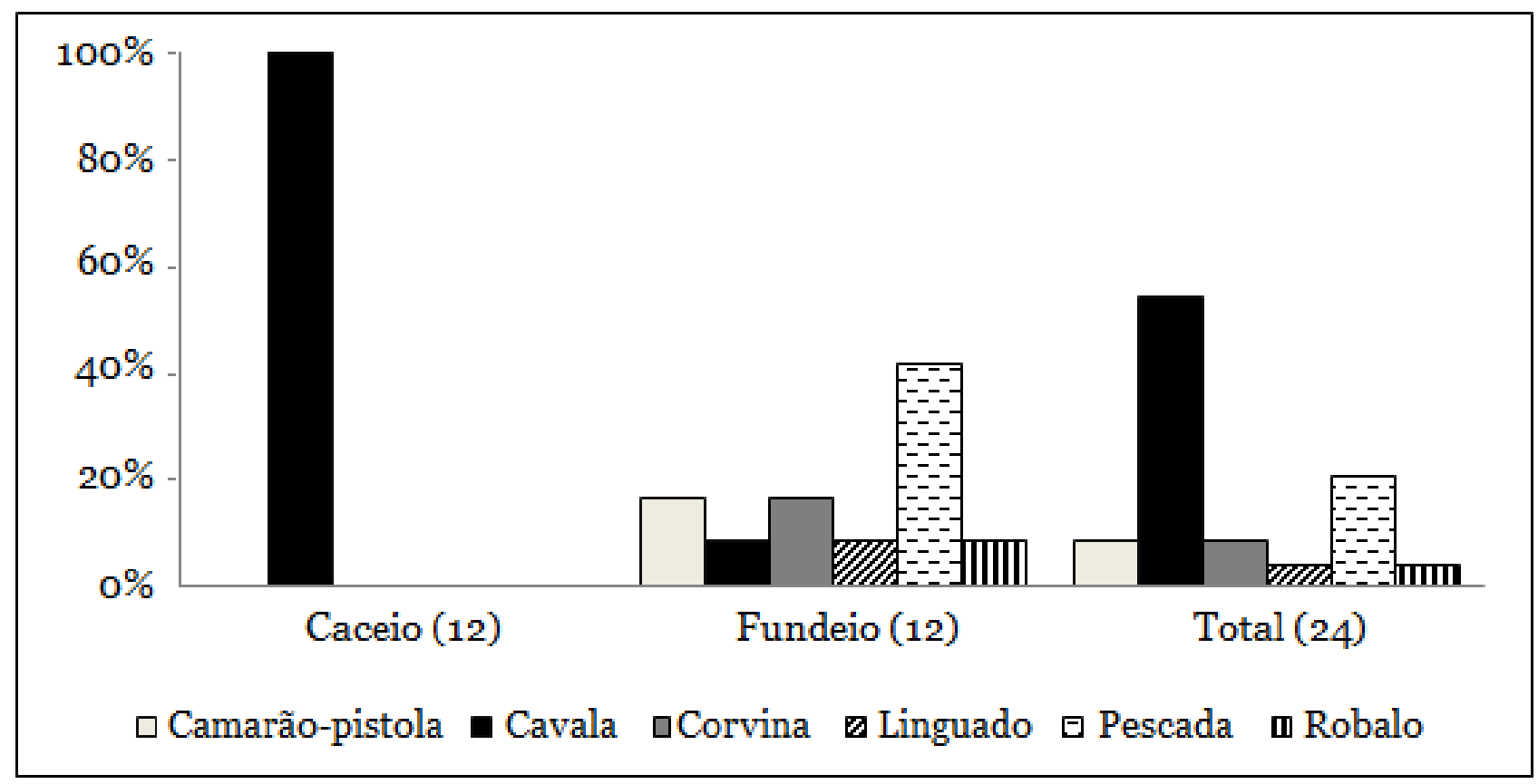

Figura 1. Frequência de ocorrência de recursos na condição de alvo em Matinhos-PR, segundo a arte de pesca com emalhe (caceio ou fundeio), no período março-outubro 2017. Entre parênteses, número de desembarques acompanhados com técnica de pesca identificada.

ca de 50\%, é inferior à diferença entre os números (5 e 23) de desembarques acompanhados, $80 \%$. No primeiro quadrimestre três desembarques tinham como alvo a pescada, e dois, o camarão-pistola. Quinze recursos eram acompanhantes, os mais frequentes betara
(18\% das observações) e pescada, cangulo, Stellifer spp, lula e siri-azul, Callinectes danae (9\%) (Tabela 5). No segundo quadrimestre os alvos foram cavala (61\% dos desembarques), corvina (22\%), pescada (9\%) e linguado e robalo (4\%). Os acompanhantes mais frequentes

Tabela 3. Recursos mais registrados como bycatch em desembarques da pesca com emalhe de março a outubro de 2017, em Matinhos-PR, segundo o alvo. ( ): desembarques observados com tal alvo. Indicam-se hábitos pelágico e demersal, conforme Tabela 2.

\begin{tabular}{|c|c|c|c|c|c|c|c|}
\hline \multirow{3}{*}{\multicolumn{2}{|c|}{ Bycatch }} & \multicolumn{6}{|c|}{ Alvo } \\
\hline & & \multicolumn{3}{|l|}{ Pelágico } & \multicolumn{3}{|c|}{ Demersais } \\
\hline & & Cavala (14) & $\begin{array}{c}\text { Pescada } \\
(8)\end{array}$ & $\begin{array}{c}\text { Corvina } \\
\text { (2) }\end{array}$ & $\begin{array}{c}\text { Robalo } \\
\text { (1) }\end{array}$ & $\begin{array}{c}\text { Linguado } \\
\text { (1) }\end{array}$ & $\begin{array}{l}\text { Camarão- } \\
\text { pistola (2) }\end{array}$ \\
\hline Pelágicos & $\begin{array}{l}\text { Espada } \\
\text { Tainha } \\
\text { Gordinho }\end{array}$ & $\sqrt{ }$ & & $\sqrt{ }$ & $\begin{array}{l}\sqrt{ } \\
\sqrt{ } \\
\end{array}$ & & \\
\hline $\begin{array}{l}\text { Demer- } \\
\text { sais }\end{array}$ & $\begin{array}{l}\text { Corvina } \\
\text { Porco } \\
\text { Pescada } \\
\text { Betara } \\
\text { Robalo } \\
\text { Camarão- } \\
\text { pistola } \\
\text { Linguado } \\
\text { Bagre } \\
\text { Cangulo } \\
\text { Cação } \\
\text { Lula } \\
\end{array}$ & $\begin{array}{l}\sqrt{ } \\
\sqrt{ }\end{array}$ & $\begin{array}{l}\sqrt{ } \\
\sqrt{ } \\
\sqrt{ }\end{array}$ & $\sqrt{ }$ & $\begin{array}{l}\sqrt{ } \\
\sqrt{ }\end{array}$ & $\sqrt{ }$ & $\begin{array}{l}\sqrt{ } \\
\sqrt{ }\end{array}$ \\
\hline
\end{tabular}


Tabela 4. Frequência de ocorrência \% dos recursos registrados como bycatch em redes de caceio (12 observações), fundeio (12 observações) e total $(28$, caceio + fundeio indistintamente). $\mathrm{N}$ : número total de ocorrências dos recursos, todas as observações reunidas.

\begin{tabular}{|c|c|c|c|}
\hline Recurso & $\underset{\%}{\text { Fundeio }}$ & $\begin{array}{c}\text { Caceio } \\
\%\end{array}$ & $\begin{array}{c}\text { Total } \\
\%\end{array}$ \\
\hline Corvina & 10,3 & 15,2 & 10,7 \\
\hline Peixe-espada & 5,2 & 19,6 & 10,7 \\
\hline Pescada & 8,6 & 8,7 & 8,2 \\
\hline Robalo & 5,2 & 8,7 & 7,4 \\
\hline Betara & 8,6 & 2,2 & 6,6 \\
\hline Peixe-porco & 1,7 & 13,0 & 5,7 \\
\hline Cação-rola-rola & 5,2 & 6,5 & 4,9 \\
\hline Cangulo & 6,9 & 0,0 & 4,1 \\
\hline Gordinho & 6,9 & 0,0 & 4,1 \\
\hline Vermelho & 1,7 & 6,5 & 4,1 \\
\hline Linguado & 5,2 & 0,0 & 3,3 \\
\hline Bagre & 3,4 & 2,2 & 2,5 \\
\hline Oveva & 5,2 &, 0 & 2,5 \\
\hline Raia & 3,4 & 0,0 & 2,5 \\
\hline Salteira & 0,0 & 4,3 & 2,5 \\
\hline Camarão-pistola & 1,7 & , & 2,5 \\
\hline Cavala & 3,4 &, 0 & 1,6 \\
\hline Lula & 4 & , & 1,6 \\
\hline Siri-azul & 3,4 & ,o & 1,6 \\
\hline Anchova & 0,0 & 2,2 & 1,6 \\
\hline Maria-luiza & 7 & 0,0 & 1,6 \\
\hline Peixe voador & 1,7 & 0,0 & 1,6 \\
\hline Sardinha & 0,0 & 2,2 & 1,6 \\
\hline Barana & 0,0 & 2,2 & 0,8 \\
\hline Camarão-sete-barbas & 1,7 & 0,0 & 0,8 \\
\hline Carapeba & 1,7 & 0,0 & 0,8 \\
\hline Caratinga & 0,0 & 2,2 & 0,8 \\
\hline Escrivão & 1,7 & 0,0 & 0,8 \\
\hline Peixe-galo & 0,0 & 2,2 & 0,8 \\
\hline Tainha & 1,7 & 0,0 & 0,8 \\
\hline Tubarão-martelo & 0,0 & 2,2 & 0,8 \\
\hline TOTAL \% & 100,0 & 100,0 & 100,0 \\
\hline $\mathbf{N}$ & 58 & 46 & 122 \\
\hline
\end{tabular}

foram peixe-espada e corvina ( $12 \%$ do total), pescada (8\%), robalo (8\%), peixe-porco (7\%) e cação (6\%) (Tabela 5).

Pescadores relataram que os meses frios são mais favoráveis para a pesca com emalhe, entretanto em 2017 as capturas com emalhe foram reportadas como sendo mais fracas que nos mesmos meses de anos anteriores. Com isso, alguns pescadores mudaram para pescaria de arrasto para camarão.
Tabela 5. Recursos na condição de bycatch na pesca com emalhe de Matinhos-PR segundo o período de estudo, ano 2017. ( ): total de desembarques; n: desembarques em que o recurso foi registrado; \%: participação relativamente à soma de ocorrência dos recursos.

\begin{tabular}{|c|c|c|c|c|}
\hline \multirow{2}{*}{ Bycatch } & \multicolumn{2}{|c|}{$\begin{array}{l}\text { Março- } \\
\text { junho (5) }\end{array}$} & \multicolumn{2}{|c|}{$\begin{array}{c}\text { Julho- } \\
\text { outubro (23) }\end{array}$} \\
\hline & $\mathbf{n}$ & $\%$ & $\mathbf{n}$ & $\%$ \\
\hline Betara & 4 & $\begin{array}{r}18, \\
0\end{array}$ & 4 & 4,0 \\
\hline Pescada & 2 & 9,0 & 8 & 8,0 \\
\hline Cangulo & 2 & 9,0 & 3 & 3,0 \\
\hline Siri-azul & 2 & 9,0 & & \\
\hline Lula & 2 & 9,0 & & \\
\hline Corvina & 1 & 5,0 & 12 & 12,0 \\
\hline Peixe-espada & 1 & 5,0 & 12 & 12,0 \\
\hline Robalo & 1 & 5,0 & 8 & 8,0 \\
\hline Raia & 1 & 5,0 & 2 & 2,0 \\
\hline Anchova & 1 & 5,0 & 1 & 1,0 \\
\hline Camarão-pistola & 1 & 5,0 & 1 & 1,0 \\
\hline Cavala & 1 & 5,0 & 1 & 1,0 \\
\hline Camarão-branco & 1 & 5,0 & & \\
\hline $\begin{array}{l}\text { Camarão-sete- } \\
\text { barbas }\end{array}$ & 1 & 5,0 & & \\
\hline Oveva & 1 & 5,0 & & \\
\hline Peixe-porco & & & 7 & 7,0 \\
\hline Cação-rola-rola & & & 6 & 6,0 \\
\hline Gordinho & & & 5 & 5,0 \\
\hline Vermelho & & & 5 & 5,0 \\
\hline Linguado & & & 4 & 4,0 \\
\hline Bagre & & & 3 & 3,0 \\
\hline Salteira & & & 3 & 3,0 \\
\hline Maria-luiza & & & 2 & 2,0 \\
\hline Oveva & & & 2 & 2,0 \\
\hline Peixe-voador & & & 2 & 2,0 \\
\hline Sardinha & & & 1 & 1,0 \\
\hline Barana & & & 1 & 1,0 \\
\hline Carapeba & & & 1 & 1,0 \\
\hline Caratinga & & & 1 & 1,0 \\
\hline Escrivão & & & 1 & 1,0 \\
\hline Peixe-galo & & & 1 & 1,0 \\
\hline Tainha & & & 1 & 1,0 \\
\hline Tubarão-martelo & & & 1 & 1,0 \\
\hline TOTAL & 22 & $\begin{array}{r}100, \\
0 \\
\end{array}$ & 100 & $\begin{array}{r}100, \\
0 \\
\end{array}$ \\
\hline
\end{tabular}

Informou-se que a despesca, com ou sem retirada das redes, pode ocorrer no mesmo dia ou no dia seguinte, dependendo da época do ano e temperatura da água, ou ante a perspectiva de condições meteorológicas adversas. 
Este conjunto de fatores pode levar à diminuição da qualidade do pescado (danos sanitários ou dilaceração física por ataque de predadores). Os pescadores relatam que, quando possível, as partes intactas são comercializadas, caso contrário, são descartadas no mar no momento da despesca. Além disso, indivíduos cuja comercialização é proibida ou que estão abaixo do tamanho mínimo de captura definido por lei, a genuína captura incidental sensu INIMPA/MMA n 10/2011 (Brasil, 2011), também são descartados.

\section{Discussão}

O número de recursos registrados, 33 , é inferior ao previamente conhecido na pesca com emalhe na região, no mínimo 45 (Afonso, 2016). Tal redução pode ser consequência, em parte, do tempo de observações, que compreendeu apenas 2/3 do ano. Mesmo que no quadrimestre novembro-fevereiro não sejam esperadas diferenças de técnicas de emalhe em relação às do período março-outubro (exceto, conforme Chaves \& Robert, 2003; Afonso, 2016, quanto ao tamanho da malha), a variedade de recursos registradas numa pescaria tende a aumentar com o esforço de observações, aqui limitadas a 28. Além disso, outras duas técnicas que utilizam emalhe na região, o caracol e o cerco (Loyola e Silva et al., 1977; Chaves \& Robert, 2003; Andriguetto-Filho et al., 2006; Afonso, 2016), não foram observadas neste estudo, mais um fato a explicar por que nem todos os recursos habituais na pesca com emalhe foram registrados. Apesar das limitações, e mesmo o esforço de campo tendo diferido entre os dois quadrimestres, com desequilíbrio no número de desembarques acompanhados, a variedade de recursos - alvo ou não - manteve -se alta em todo o período de estudo.

Bycatch no emalhe afeta tanto caceio como fundeio, com significativa coincidência entre as técnicas, pois um terço dos recursos foi bycatch em ambas. Porém, 15 recursos foram bycatch exclusivamente no fundeio, enquanto no caceio, sete. $\mathrm{O}$ menor número de gêneros registrados deve-se ao fato de o caceio ser pes- ca dirigida a cardumes de superfície (Chaves \& Robert, 2003), menos acessível a recursos demersais, que são os mais variados na região. De fato, caceio foi associado ao alvo cavala, recurso pelágico, enquanto fundeio foi associado a maior número de alvos, todos demersais.

Bycatch de recurso pelágico em redes com alvo demersal pode ocorrer no recolhimento do equipamento. O caráter episódico dessas capturas, associado ao baixo número de observações em meses de outono, explica a ausência de tainhas como bycatch de caceio, cujos lances são dirigidos a cardumes de cavalas. $\mathrm{Na}$ região as operações para captura de tainhas dão-se majoritariamente por cerco (Andriguetto-Filho et al., 2006). Quanto ao inverso, bycatch demersal em alvo pelágico, é parcialmente explicado pela altura das redes, superior a 20 metros. Se, por um lado, verticalidade e distensão absolutas da panagem não são esperadas, por esta sofrer influência da corrente e, durante caceio, do movimento da embarcação, por outro, mesmo que a altura operacional não alcance a altura nominal, a coluna de abrangência de uma rede de emalhe mostrou-se expressiva. Adicionalmente, operações de caceio são realizadas próximas à costa, também, e neste caso a rede tende a cobrir a totalidade da coluna d'água.

Ocorrência de elasmobrânquios maior em inverno-primavera que em verão-outono foi também reportada na região por Costa \& Chaves (2006). Grupo com delicado status de conservação (ICMBio, 2016), a proibição da captura é de mais difícil cumprimento quando indivíduos ocorrem como bycatch. Ademais, pesquisas sobre captura incidental costumam negligenciar descartes havidos a bordo, matéria contemplada por Schroeder et al. (2014) no emalhe industrial da corvina no Sudeste/Sul do Brasil. Mesmo se descartados vivos, os indivíduos estão sob nível de estresse e ferimentos que podem levar à morte ou deixar sequelas (Davis, 2002), gerando baixas ignoradas pela estatística.

Constatou-se que parte do bycatch é aproveitada na região. Mesmo assim, é grande a geração de resíduos de pesca, estimada no 
local de estudo em meia tonelada diária, indivíduos inteiros ou partes, bycatch e alvos, emalhe e arrasto reunidos (Chaves \& Vink, 2017).

Todos os recursos-alvo foram também detectados como bycatch em pelo menos um desembarque. A constatação alerta que se a gestão pesqueira se detiver em petrechos ou épocas do ano sob a referência dos recursosalvo, exclusivamente, ou de espécies-bandeira, como as ditas carismáticas, os impactos a que recursos pesqueiros variados encontram-se sujeitos em pescarias nas quais eles não são alvo, legalizadas à luz do recurso-alvo, estarão sendo negligenciados. Em vista disso, medida mais eficaz para a explotação sustentável pode resultar da soma de dois elementos: um, conforme recomendação de IBAMA/CEPSUL (2006), a definição de áreas de exclusão de pesca, o que contempla conjuntamente alvo e não-alvo; e outro, para definição de tais áreas, a consideração do grau de incidência de fauna acompanhante como um todo, incluindo recursos pesqueiros em suas ecofases mais sensíveis.

\section{Referências Bibliográficas}

AFONSO, M. G. 2016. Dinâmicas da pesca de emalhe em Matinhos-PR: implicações para a conservação dos recursos pesqueiros. Curitiba, 47p. (Dissertação de Mestrado - Zoologia UFPR.)

ALVERSON, D. L., FREEBERG, M. H., POPE, J. G. \& MURAWSKI, S. A. 1994. A global assessment of fisheries bycatch and discards. FAO Fisheries Technical Paper. No 339. Rome, FAO: 233

ANDRIGUETTO-FILHO, J. M., CHAVES, P. T., SANTOS, C. \& LIBERATI, S. A. 2006. Diagnóstico da pesca no litoral do estado do Paraná. In ISAAC, V. J.; HAIMOVICI, M., MARTINS, S. A. \& ANDRIGUETTO-FILHO, J. M. A pesca marinha e estuarina do Brasil no início do século XXI: recursos, tecnologias, aspectos socioeconômicos e institucionais. Belém. UFPA: 186p.

BARBANTI, B., CAIRES, R. \& MARCENIUK, A. P. 2013. A ictiofauna do Canal de Bertioga,
São Paulo, Brasil. Biota Neotr., 13(1): 267291.

BRASIL, 2011. Instrução Normativa MPA/ MMA no 10/2011. Aprova as normas gerais e a organização do sistema de permissionamento de embarcações de pesca para acesso e uso sustentável dos recursos pesqueiros, com definição das modalidades de pesca, espécies a capturar e áreas de operação permitidas.

CARDOSO, L.G., BUGONI, L., MANCINI, L., HAIMOVICI, M. 2011 Gillnet fisheries as a major mortality factor of Magellanic penguins in wintering areas. Mar Poll Bull 62:840844.

CHALEGRE, K. Q. T. 2008. Fauna bêntica do infralitoral e alimentação natural de Callinectes danae Smith 1869 (Crustacea, Portunidae) nos estuários dos rios Botafogo e Carrapicho, Pernambuco, Brasil. Recife, 107p. (Dissertação de Mestrado, Oceanografia, UFPE.)

CHAVES, P. T. \& ROBERT, M. C. 2003. Embarcações, artes e procedimentos da pesca artesanal no litoral sul do estado do Paraná, Brasil. Atlântica, Rio Grande, 25(1): 53-59.

CHAVES, P. T. \& VINK, J. M. 2017. Rejeitos da atividade pesqueira no litoral do Paraná: gestão atual e potencial para destinação alternativa. Revista CEPSUL - Biodiversidade e Conservação Marinha, 6: 1-10.

CORTÉS, E., AROCHA, F., BEERKIRCHER, L., CARVALHO, F., DOMINGO, A., HEUPEL, M., HOLTZHAUSEN, H., SANTOS, M. N., RIBERA, M. \& SIMPFENDORFER, C. 2010. Ecological risk assessment of pelagic sharks caught in Atlantic pelagic longline fisheries. Aquat. Living Resour. 23, 25-34.

COSTA, L. \& CHAVES, P. T. C. 2006. Elasmobrânquios capturados pela pesca artesanal na costa sul do Paraná e norte de Santa Catarina, Brasil. Biota Neotr., 6(3): bno2706032006.

DAVIS, M. W. 2002. Key principles for understanding fish bycatch discard mortality. Can. J. Fish. Aquat. Sci., 59: 1834-1843. 
EAYRS, S. 2007. A guide to bycatch reduction in tropical shrimp-trawl fisheries. Revised edition. Roma, FAO. 108 p.

FAGUNDES-NETTO, E. B., GAELZER, L. R., COUTINHO, R. \& ZALMON, I. R. 2011. Influence of a ship wreck on a nearshore-reef fish assemblages off the coast of Rio de Janeiro, Brazil. Lat. Am. J. Aquat. Res., 39(1): 103-116.

FEITOZA, B. M., ROSA, R. S. \& ROCHA, L. A. 2005. Ecology and zoogeography of deep reef fishes in northeastern Brazil. Bull. of Mar. Science, 76(3): 725-742.

GUIMARÃES, F.J. 2009. Biologia do camarão sete barbas, Xiphopenaeus kroyeri (Heller, 1862) (Crustacea, Decapoda, Penaeidae) e aspectos da sua pesca na Reserva Extrativista Marinha do Corumbau, BA. João Pessoa, 65p. (Tese de Doutorado Ciências Biológicas, UFPB.)

HAIMOVICI, M., VASCONCELLOS, M., KALIKOSKI, D. C., ABDALAH, P., CASTELLO, J. P. \& HELLEBRANDT, D. 2006. Diagnóstico da pesca no litoral do estado do Rio Grande do Sul. In ISAAC, V.J., MARTINS, A.S., HAIMOVICI, M. \& ANDRIGUETTO-FILHO, J.M. (orgs.). A pesca marinha e estuarina do Brasil no século XXI: recursos, tecnologias, aspectos socioeconômicos e institucionais. Belém, Ed. UFPA: 141-156.

IBAMA/CEPSUL 2006. Relatório da Reunião Técnica sobre a Pesca de Emalhe no Litoral Brasileiro. Itajaí, 28/8 a 01/9/2006.48p.

ICMBio 2016. Avaliação do risco de extinção dos elasmobrânquios e quimeras no Brasil: 20102012. CEPSUL. Itajaí, 66p.

LENTA, R. \& SQUIRES, R. 2017. Reducing marine mammal bycatch in global fisheries: an economics approach. Deep-Sea Research Part II 140: 268-277.

LOYOLA e SILVA, J., TAKAI, M. E. \& CASTRO, R.M.V. 1977. A pesca artesanal no litoral paranaense. Acta Biol. Par., Curitiba, 6: 95-12.

MAGRO, M., CERGOLE, M. C. \& ROSSIWONGTSCHOWSKI, C. L. B. 2000. Síntese de conhecimentos dos principais recursos pesqueiros costeiros potencialmente explotáveis na costa sudeste-sul do Brasil: peixes. MMA/CIRM, Brasília.

MENEZES, N.A. \& FIGUEIREDO, J.L. 1980. Manual de Peixes Marinhos do Sudeste do Brasil. Museu de Zoologia, USP, São Paulo, 96p.

MULATO, I. P., CORRÊA, B. \& VIANNA, M. 2015. Distribuição espaço-temporal de $M i$ cropogonias furnieri (Perciformes, Sciaenidae) em um estuário tropical no sudeste do Brasil. Bol. Inst. Pesca, São Paulo, 41(1): 1-18.

NOAA 2007. Magnuson-Stevens Fishery Conservation and Management Act. Washington, U.S. Department of Commerce, 170p.

PAIVA, M. P. \& MOTTA, P. C. S. 2000. Cardumes da sardinha-verdadeira, Sardinella brasiliensis (Steindachner), em águas costeiras do estado do Rio de Janeiro, Brasil. Revta bras. Zool., 17 (2): 339-346

PINA, J. V. \& CHAVES, P. T. 2009. Incidência da pesca de arrasto camaroeiro sobre peixes em atividade reprodutiva: uma avaliação no litoral norte de Santa Catarina, Brasil. Attântica, Rio Grande, 31(1) 99-106.

ROCHA, G. R. A. \& ROSSI-WONGTSCHOWSKI, C. L. D. B. 1998. Demersal fish community on the inner shelf of Ubatuba, southeastern Brazil. Revta bras. Oceanogr., 46(2): 93-109.

SANTOS, M. C. F., PEREIRA, J. A. \& IVO, C. T. C. 2004. Sinopse de informações sobre a biologia e pesca do camarão-branco, Litopenaeus schimitti (Burkenroad, 1936) (Crustacea, Decapoda, Penaeidae), no nordeste do Brasil. Bol. Técn. Cient. CEPENE, v. 12(1): 149-185.

SCHROEDER, R., PIO, V. M., BAIL, G. C., LOPES, F. R. A. \& WAHRLICH, R. 2014. Análise Espaço-Temporal da Composição da Captura da Pesca com Emalhe de Fundo no Sudeste/Sul do Brasil. Bol. Inst. Pesca, S. Paulo, 4O(3): 323-353.

SOUZA, L. M. \& CHAVES, P. T. 2007. Atividade reprodutiva de peixes (Teleostei) e o defeso da pesca de arrasto no litoral norte de Santa 
Catarina, Brasil. Revta bras. Zool., 24(4): 1113 $-1121$.

ZALESKI, T. 2010. Ciclo de vida e ecologia da lula Lolliguncula brevis na Armação de Itapocoroy, Santa Catarina, Brasil. Curitiba, 188p. (Tese de Doutorado Zoologia UFPR.) 